\title{
Modelos Latentes e Slopes Randômicos para Análise de Moderação e Mediação
}

\author{
Felipe Valentini ${ }^{1}$ \\ Universidade São Francisco, Campus Swift, Campinas-SP, Brasil \\ Luciana Mourão \\ Universidade Salgado de Oliveira, Niterói-RJ, Brasil \\ Vithor Rosa Franco \\ Universidade de Brasília, Brasília-DF, Brasil
}

\section{RESUMO}

Modelos complexos de mediação e moderação são fundamentais para a compreensão dos fenômenos psicológicos. No entanto, ao testar esse tipo de modelagem, a estrutura e a imprecisão dos instrumentos podem influenciar a estimação dos efeitos entre as variáveis. Assim, modelos de mediação e moderação que corrigem os parâmetros com as estimativas de erros são fundamentais. O objetivo deste estudo é comparar o desempenho de três modelagens de mediação e moderação: slopes randômicos, interações latentes e path analysis. Os três modelos foram comparados tanto com o uso de dados simulados quanto com o uso de dados reais. Os resultados apontaram que, quando a variância dos slopes é pequena, os modelos de slopes randômicos e de interações latentes são praticamente equivalentes. Já o modelo de path analysis obteve bom desempenho para recuperar os efeitos de moderação, mas subestimou consideravelmente os efeitos diretos e indiretos da mediação. Discutem-se melhores práticas de testagem de hipóteses com efeitos complexos e sugere-se o uso da modelagem latente para testes de mediação e moderação.

Palavras-chave: moderação; mediação; interações latentes; slopes randômicos; path analyses.

\section{ABSTRACT - Latent Models and Random Slopes for Testing Moderation and Mediation}

Complex models, such as mediation and moderation, are essential for comprehending psychological variables. Nevertheless, during the testing of such models, the structure and reliability of the instruments might affect the estimated relationship between variables. Thus, models of mediation and moderation that correct the parameters yielding error estimates are fundamental. The objective of this study is to compare the performance of three Structural Equation Modeling models that include mediation and moderation effects: Random slopes, latent interactions and path analysis. The three models were compared using both simulated and real data. The results showed that when the variance contained in the slopes is small, both the random slopes model and the latent interaction model are equivalently good. The path analysis model, on the other hand, performs well to recover the effects of moderation, but it underestimates the direct and indirect effects of mediation. Best practices for testing hypotheses with complex effects are discussed, and we suggest using latent models for testing mediation and moderation.

Keywords: moderation; mediation; latent interactions; random slopes; path analysis.

\section{RESUMEN - Modelos Latentes y Slopes Aleatorios para Análisis de Moderación y Mediación}

Los modelos complejos de mediación y moderación son claves para la comprensión de los fenómenos psicológicos. No obstante, al probar este tipo de modelado, la estructura y la inexactitud de los instrumentos pueden influir en la estimación de los efectos de las relaciones entre las variables. De modo que, los modelos de mediación y moderación que corrigen los parámetros con las estimativas de error, son imprescindibles. El objetivo de este estudio es comparar el desempeño de tres modelos de análisis de mediación y moderación: slopes aleatorios, interacción latente y path analysis. Los tres modelos fueron comparados utilizando datos simulados y reales. Los resultados indicaron que, cuando la variancia de los slopes es pequeña, los modelos de slopes aleatorios e interacción latente son prácticamente equivalentes. Por otra lado, el modelo de path analysis obtuvo un desempeño satisfactorio para recuperar los efectos de moderación, a pesar de que subestimó notablemente los efectos directos y indirectos de la mediación. Se discuten mejores prácticas para los testeos de hipótesis con efectos complejos, y se sugiere el uso del modelo latente para los análisis de mediación y moderación.

Palabras clave: moderación; mediación; interacción latente; slopes aleatorio; path analysis.

A maior parte das pesquisas em ciências humanas e sociais envolve a avaliação de relações entre variáveis, com crescente interesse nos modelos em que uma variável medeia a relação entre outras duas variáveis (Zhao, 
Lynch, \& Chen, 2010). Da mesma forma, têm crescido as pesquisas que incluem variáveis moderadoras, uma vez que a força da relação entre duas variáveis também pode depender de uma terceira (Baron \& Kenny, 1986). Assim, mais do que investigar apenas correlações, em geral, os pesquisadores estão interessados em descobrir caminhos causais (Hayes, 2009). Não é recente, portanto, o argumento de que, para a construção de teorias mais robustas sobre processos cognitivos e comportamentais, é necessário o uso de modelos que explicam mais do que relações diretas entre variáveis independentes e dependentes (Baron \& Kenny, 1986; Cohen \& Cohen, 1983; Fiske, Kenny, \& Taylor, 1982).

Em algumas situações, há interesse em investigar, inclusive, a operação simultânea entre variáveis moderadoras e mediadoras (Donaldson, 2001, Zhao et al., 2010). Contudo, grande parte das pesquisas em Psicologia ainda utiliza regressões ou outros métodos que assumem que os escores dos indivíduos são observados e livres de erros de mensuração (Aguinis, Edwards, \& Bradley, 2017; Cheung \& Lau, 2017). Como tal pressuposto é pouco plausível, a escolha por tais métodos resulta em perda de precisão nas estimações. Diante do exposto, o uso de modelos latentes para mediação e moderação representaria uma solução para conciliar aspectos relativos aos erros de mensuração e à testagem de modelos mais complexos de relações entre variáveis (Bollen, 1989).
Assim, o objetivo do presente artigo é comparar o desempenho de três modelos de modelagem por equações estruturais que incluem efeitos de mediação e moderação: slopes randômicos, interações latentes e path analysis (análise de caminhos). Para possibilitar a compreensão dessas diferentes aplicações, serão apresentados a seguir alguns conceitos básicos que fundamentam o presente estudo, abordando as definições e alguns desafios relativos a esses modelos.

\section{Mediação: A Testagem de Efeitos Indiretos}

O conceito de mediação não é novo (Hyman, 1955; Rozeboom, 1956) e embora modelos com mediação ainda sejam pouco utilizados, há um interesse crescente, sobretudo pela possibilidade de explicar relações causais sequenciais. Assim, em um modelo mediacional clássico, tem-se que a variável independente (VI) é preditora da variável mediadora (VMed) e esta, por sua vez, prediz a variável dependente (VD), de tal sorte que quando a VMed está presente na equação de regressão, ela elimina ou reduz a magnitude do relacionamento entre a VI e a VD (Abbad \& Torres, 2002; MacKinnon, Lockwood, Hoffman, West, \& Sheets, 2002). Em estatística, o efeito da VI sobre a VD, quando mediado pela VMed, é chamado de efeito indireto (Cohen \& Cohen, 1983). Tais relações são ilustradas na Figura 1.

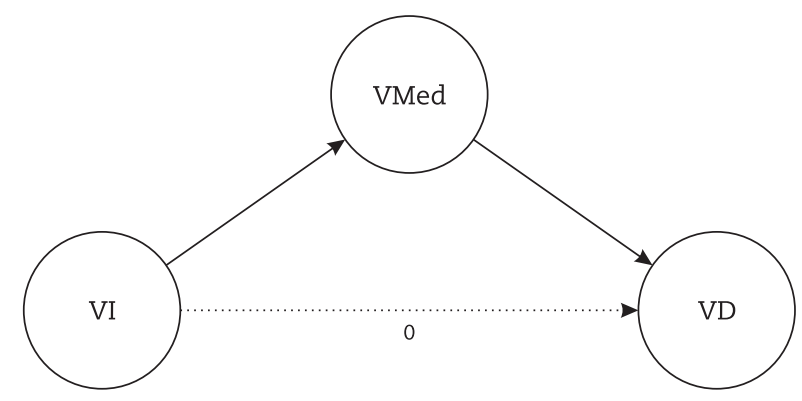

Figura 1. Modelo de mediação com efeito indireto entre VI e VD, e efeito direto não diferente de 0

Assim, as análises mediacionais são usadas para avaliar o grau em que uma variável é intermediária na sequência causal entre um preditor e uma variável dependente (Morgan-Lopez \& MacKinnon, 2006). Baron e Kenny (1986) apresentaram, contudo, três condições que precisariam ser atendidas para uma variável funcionar como mediadora, a saber: (a) variações na VI implicam variações significativas na VMed; (b) variações na VMed implicam variações significativas na VD; e (c) quando se introduz a VMed no modelo, a relação anteriormente significativa entre a VI e a VD deixa de existir ou torna-se mais fraca. A presença dessas condições embasou a tipologia da modelagem de mediação, proposta por Zhao, Lynch e Chen (2010), que prevê: (a) mediação complementar - o efeito mediado e o efeito direto existem e apontam na mesma direção; (b) mediação competitiva - o efeito mediado e o efeito direto existem e estão em direções opostas; (c) mediação apenas indireta - o efeito mediado existe, mas o efeito direto não; (d) não mediação direta - o efeito direto está presente, mas inexiste efeito indireto; e (e) não-mediação sem efeito - não existe nem efeito direto, nem efeito indireto.

No que diz respeito aos testes utilizados para avaliar a mediação, os mais utilizados são regressões, path analysis e equações estruturais (MacKinnon, 2008). Nos dois primeiros, as variáveis são consideradas como observadas e, no último, além dos efeitos entre variáveis, 
são estimadas também variáveis latentes. Embora as conclusões das três modelagens tendam a ser convergentes, estudos têm indicado que os efeitos de relação entre variáveis são mais bem estimados por equações estruturais, ponderando o erro de medida (Hoyle \& Kenny, 1999; Muthén \& Asparouhov, 2015). Modelos com variáveis observadas tendem a subestimar o efeito indireto e os erros-padrão de estimativa, bem como superestimar o efeito direto entre VI e VD, o que diminui o poder de deteç̧ão da mediação (Muthén \& Asparouhov, 2015).

Além dos aspectos técnicos, Kenny (2008) ressalta a importância de um modelo teórico, que ofereça suporte aos pressupostos causais subjacentes à análise mediacional, pois a sua ausência pode conduzir a uma especificação incorreta do modelo, tornando enganosos os resultados da análise mediacional. Para além dos casos de mediação, há também interesse em modelos que envolvem variáveis moderadoras, conforme discutido na próxima seção.

\section{Moderação: Efeitos de Interação}

A relação entre duas variáveis, muitas vezes, depende de uma terceira, chamada de variável moderadora (VMod) (Baron \& Kenny, 1986; Donaldson, 2001). As variáveis moderadoras afetam a força ou a direção da relação entre duas variáveis e são tipicamente testadas com efeitos de interação, isto é, a partir de um efeito multiplicativo entre VI e VMod, ou termo de interação (Morgan-Lopez \& MacKinnon, 2006; Zhang \& Wang, 2017). Essas análises são úteis, por exemplo, para avaliar se uma relação entre duas variáveis pode diferir em direção ou em magnitude em subpopulações ou em contextos distintos (Baron \& Kenny, 1986; MorganLopez \& MacKinnon, 2006). Tais relações são ilustradas na Figura 2.

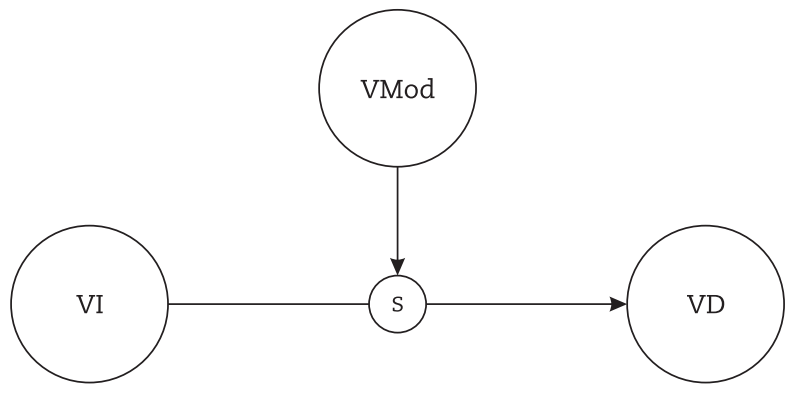

Figura 2. Modelo de moderação

Análises de moderação (ou de interação) são importantes porque a maioria dos efeitos que os pesquisadores de ciências humanas e sociais estudam são contingentes a covariantes externas, por exemplo, podem ser maiores em mulheres e menores em homens ou positivos para determinadas categorias e negativos para outras. Hayes (2013) apresenta um exemplo clássico, na área da saúde pública, no qual o nível educacional (VMod) modera o efeito da exposição à informação (VI) sobre o conhecimento de determinada temática (VD), de tal sorte que o gap de conhecimento entre aqueles que têm maior e menor escolaridade tende a aumentar se ambos têm a mesma exposição à informação.

A maneira clássica para testar a moderação envolve a interação entre as variáveis VI e VMod, configuradas como observadas (Hayes, 2013). Assim, um primeiro passo envolve a multiplicação dessas duas variáveis observadas $(\mathrm{VI} \times \mathrm{VMod})$; e no segundo passo, testa-se, por regressão múltipla (ou por path analysis), a capacidade preditiva da VI, VMod e do termo de interação (VI $\times$ VMod). Uma limitação importante dessa modelagem diz respeito à ausência de ponderação da estrutura e da consistência interna dos escores. Assim, as variáveis são assumidas como observadas e livres de erros de mensuração, o que não necessariamente se sustenta (Little, Card, Bovaird, Preacher, \& Crandall, 2007).

Para lidar com essa limitação, existem diversas técnicas, no contexto da modelagem por equações estruturais, capazes de estimar, simultaneamente, efeitos de moderação e variáveis latentes. Nessa lógica, as relações entre as variáveis são estimadas ponderando a estrutura dos instrumentos que compõem as variáveis e o respectivo erro de medida (Trautwein et al., 2012). Os modelos latentes permitem estimar erros-padrão sem viés substancial e, adicionalmente, fornece insights sobre a estrutura estocástica da interação latente (Klein \& Moosbrugger, 2000). O estudo de simulação implementado por esses autores sugere que a estimação de modelos latentes, por equações estruturais, é mais eficiente do que a estimativa realizada com técnicas tradicionais. Entre os modelos latentes para testes de moderação, cita-se a interação latente (Klein \& Moosbrugger, 2000; Klein \& Muthén, 2002; Muthén, 2012), a interação latente com indicadores ortogonalizados (Little, Bovaird, \& Widaman, 2006) e os slopes randômicos (Muthén \& Asparouhov, 2003). 
No primeiro caso, o modelo com interações latente envolve a estimação de uma variável a partir da interação dos itens dos instrumentos da VI e da VMod. Em outras palavras, os indicadores da variável de interação latente (VInt) são calculados a partir da multiplicação dos itens da VI e da VMod. Por exemplo: indicador $1=$ item 1 da VI $\times$ item 1 da VMod; indicador $2=$ item 1 da VI $\times$ item 2 da VMod; e assim sucessivamente.

Essa variável latente, estimada a partir das interações entre os itens, é utilizada no modelo com o teste de moderação. Assim, o modelo final é estimado com relações entre VI-VD, VMod-VD e VInt-VD, sendo todas variáveis configuradas como latentes. No entanto, essa modelagem não é isenta de problemas: um mesmo item (da VI ou da VMod) é utilizado para compor mais de um indicador da interação latente (veja no exemplo acima, no qual o item 1 da VI é utilizado em ambos os indicadores 1 e 2); o que gera covariância residual entre os indicadores da VInt. Uma maneira simples de resolver o problema é estimar as covariâncias residuais entre os indicadores que envolvem itens em comum (Little et al., 2006).

Além dessa solução de correções entre os resíduos, Little et al. (2006) propuseram a ortogonalização dos indicadores da VInt (modelo com interações latentes a partir de indicadores ortogonalizados). Nessa proposta, a variância compartilhada entre os itens é removida dos indicadores de interação. Após promover a interação entre os itens da VI e VMod e compor os indicadores da VInt, são realizadas regressões predizendo a VInt a partir de todos os itens da VI e da VMod. Assim, os resíduos dessas regressões contêm a variância dos indicadores (da VInt) que não é compartilhada com os itens da VI e VMod. Como esses resíduos são ortogonais aos itens da VI e da VMod, eles passam a ser utilizados para estimar a variável latente de interação (VInt) em vez dos indicadores originais. O estudo de simulação de Little et al. (2006) indicou que o modelo de interação latente (sem ortogonalização) estimou os parâmetros da moderação de maneira levemente menos enviesada se comparado ao modelo ortogonalizado (vieses de $0,1 \%$ e $3,9 \%$, respectivamente). Ainda assim, as diferenças entre os efeitos verdadeiros (simulados) e os estimados foi pequena em ambas as modelagens.

Por fim, uma maneira "elegante" de testar moderações em equações estruturais refere-se ao modelo de slopes randômicos (Muthén \& Asparouhov, 2003). Nos modelos anteriores, os efeitos das VI, VMod e VInt sobre a VD eram todos fixos (i.e., não eram consideradas variações entre os participantes). Contudo, a ideia teórica da moderação envolve uma relação entre VI e VD que não é fixa, pois supõe variação entre os participantes de acordo com uma outra variável (VMod). Assim, nada mais "sofisticado" do que permitir que o efeito da relação entre VI e VD varie entre os sujeitos (ou seja, ser um efeito randômico). Assim, o próprio efeito de slope tem média (ou intercepto) e variância estimadas. Reparem que, na
Figura 2, o efeito da VD sobre a VI é representado por um S, de slope randômico, dentro de um círculo pequeno, pois tem a sua variância estimada. Considerando que o próprio efeito (slope) passa a ter variância, ele pode, então, ser explicado por outras variáveis (VMod).

A diferença técnica entre os modelos de interação latente e o de slope randômico é concentrada na variância dos slopes, que é estimada apenas para esse último. Veja as equações:

$$
Y=\beta_{0}+\beta_{s 1} X+\varepsilon_{y}
$$

Essa é uma equação de relação entre duas varáveis (X e Y), na qual, $\mathrm{Y}=\mathrm{VD}, \beta_{0}=$ intercepto, $\beta_{s 1}=$ slope randômico entre VI e VD, $\mathrm{X}=\mathrm{VI}, \varepsilon_{y}=$ resíduo. Como o efeito da VI sobre a VD é randômico $\left(\beta_{s 1}\right)$, esse efeito também pode ser representado por uma equação:

$$
\beta_{s 1}=\beta_{0 s}+\beta_{w} W+\varepsilon_{s}
$$

$\mathrm{Na}$ qual, $\beta_{0 s}=$ intercepto (ou média) dos efeitos randômicos, $\beta_{w}=$ efeito da moderação, $\mathrm{W}=\mathrm{VMod}$; $\varepsilon_{s}=$ resíduo do slope randômico. Substituindo o efeito randômico $\left(\beta_{s 1}\right)$ da Equação 2, nos termos da Equação 1, tem-se a seguinte equação resultante do modelo de slopes randômicos:

$$
\mathrm{Y}=\beta_{0}+\beta_{0 \mathrm{~s}} \mathrm{X}+\beta_{\mathrm{w}} \mathrm{WX}+\varepsilon_{\mathrm{s}} \mathrm{X}+\varepsilon_{\mathrm{y}}
$$

Na Equação 3, é notável que o modelo de slopes randômicos também envolve uma interação entre VI e VMod (parâmetro $\varepsilon_{w} \mathrm{WX}$ ). No entanto, adiciona um parâmetro de variância dos slopes $\left(\varepsilon_{s}\right)$. Caso esse parâmetro $\left(\varepsilon_{s}\right)$ seja fixado em 0 (i.e., não há variância dos slopes para além do que é explicado pela interação WX), os modelos de slopes randômicos e de interação latente tornam-se equivalentes. Vale registrar que, nas Equações 1, 2 e 3, as matrizes e vetores dos itens que compõem as variáveis $\mathrm{Y}$, $\mathrm{X}$ e W, foram omitidas para fins de simplificação.

\section{Modelos que Integram Mediação e Moderação: Efeitos Indiretos Condicionais}

Modelos de mediação nos quais os moderadores também são examinados podem fornecer mais informações para os pesquisadores compreenderem os fenômenos que estão estudando, como nos casos em que a VMod modera o efeito da VI na VMed ou o efeito da VMed na VD (Baron \& Kenny, 1986; Donaldson, 2001; 
Morgan-Lopez \& MacKinnon, 2006). Assim, integrar moderação e mediação pode levar a insights importantes para os pesquisadores, bem como para os profissionais que atuam nas áreas de ciências humanas e sociais (Aguinis et al., 2017).

Assim, o termo "mediação moderada" foi cunhado por James e Brett (1984), para modelos de mediação envolvendo relações que requerem a adição de um moderador. Do ponto de vista conceitual, a mediação moderada ocorre quando a força de um efeito indireto depende do nível de alguma variável, ou seja, quando as relações indiretas são contingentes ao nível de um determinado moderador (Preacher, Rucker, \& Hayes, 2007). A Figura 3 traz um exemplo de mediação moderada.

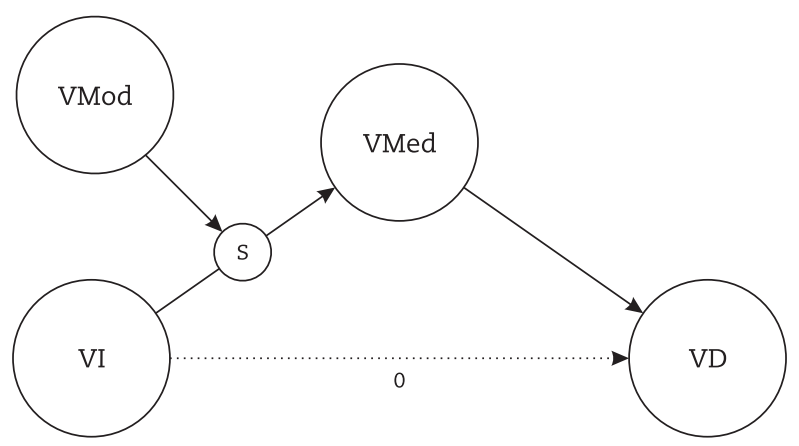

Figura 3. Modelo de mediação e moderação

No exemplo da Figura 3, o efeito entre VI e VD é intermediado pelo VMed, e esse efeito indireto, por sua vez, é modulado pela VMod. Variações desse modelo também podem ser testadas, por exemplo: moderação dos efeitos diretos entre VMed-VD ou dos efeitos entre VI e VD. Embora essas variações possam receber diferentes termos como moderação mediada e mediação moderada, a depender de onde a VMod é posicionada (Baron \& Kenny, 1986; Donaldson, 2001; Preacher et al., 2007; Wu \& Zumbo, 2008), as diferenças são sutis e fontes de confusão. Assim, para evitar equívocos, sugere-se o uso do termo efeitos indiretos condicionais (Preacher et al., 2007). Tal terminologia pode ser justificada ao se levar em conta que o efeito de interesse é um efeito indireto (mediação), que é potencialmente condicional ao valor de uma ou mais variáveis moderadoras.

Para além das questões conceituais, os modelos complexos de mediação e moderação também sofrem de problemas metodológicos que comprometem a sua utilidade. Edwards e Lambert (2007) apontam três situações em que a combinação de moderação e mediação são feitas de modo inadequado. Na primeira, a moderação e a mediação são analisadas separadamente, e os resultados dessas análises são interpretados em conjunto para descrever os efeitos combinados de moderação e mediação. Na segunda, a amostra é dividida em subgrupos que representam diferentes níveis da variável moderadora, e a mediação é examinada dentro de cada subgrupo. E, na terceira, o procedimento de etapas causais para avaliar a mediação é adaptado para incorporar variáveis moderadoras, testando se um efeito moderador já significativo não é mais significativo após o controle de uma variável mediadora. Cada uma dessas situações de análise pode conduzir a diferentes erros de interpretação dos resultados, sendo recomendável, portanto, que sejam realizadas análises simultâneas de moderação e mediação a partir da modelagem por equações estruturais.

Considerando a contribuição que modelos de mediação e moderação têm para a explicação de relação entre variáveis na Psicologia, os pesquisadores têm procurado formas mais adequadas para se testar os efeitos de variáveis moderadoras e mediadoras (Cheung \& Lau, 2008; Morgan-Lopez \& MacKinnon, 2006). Aguinis et al. (2017) realizaram uma pesquisa para investigar problemas metodológicos relacionados a moderação e mediação a partir de uma revisão de artigos publicados entre 2005 e 2014. Esse estudo apontou para a desconsideração dos erros de mensuração como o problema mais recorrente, com ocorrência de 88,7\% dentre os 62 artigos de mediação analisados. Tal resultado aponta para a necessidade de se usar modelos que possam corrigir esse tipo de problema.

Nesse contexto, Cheung e Lau (2017) compararam a performance de modelos latentes (com a ponderação do erro de medida) e de regressões (com variáveis configuradas como observadas) na recuperação de parâmetros de dados simulados para contemplar mediações moderadas. Por um lado, os resultados indicaram que os modelos de regressões subestimam os efeitos de mediação e moderação. Por outro, modelos latentes estimam os efeitos entre as variáveis de maneira muito próxima aos parâmetros verdadeiros (simulados), indicando pouco viés de estimação.

Apesar de a superioridade do uso de modelos com variáveis latentes para a testagem de hipóteses moderacionais e mediacionais já ter sido apontada por Cheung 
e Lau (2017), os modelos de interações latentes e slopes randômicos não foram comparados quanto a suas performances em modelos de mediação moderada. O presente estudo apresenta comparações, com uso de dados simulados e reais, que são úteis para ilustrar as diferenças entre as modelagens de mediação moderada (ou de efeitos indiretos condicionados).

O estudo específico com dados simulados teve como objetivo avaliar a qualidade da recuperação dos parâmetros de um modelo de mediação moderada, quando os slopes são randômicos na população. Em outras palavras, buscou-se avaliar qual modelagem é mais indicada para dados nos quais o efeito de uma VI sobre uma VMed difere significativamente em função de determinada condição dos participantes.

\section{Método}

\section{Exemplo com Dados Simulados: Recuperação de Parâmetros}

Para atender aos objetivos desta pesquisa, os dados foram simulados para contemplar efeitos randômicos (com variância diferente de zero) entre VI e VMed. O modelo configurado foi de efeitos indiretos condicionados (mediação moderada) com apenas uma VMed entre a relação de uma VI e uma VD, bem como uma VMod entre a VI e a VMed (conforme exemplo da Figura 3). Foram simuladas duas condições de tamanho de efeito para esse modelo: (a) efeitos moderados, com moderação igual 0,40 e mediação (efeito indireto) igual 0,20; (b) efeitos fracos, com moderação igual a 0,10 e mediação igual a 0,05 . O modelo populacional (modelo verdadeiro de base para a simulação dos dados) contemplou slopes randômicos com variância estatisticamente significativa. Com isso, buscou-se abarcar dois cenários comuns em Psicologia: efeitos de moderação fraco e efeitos de moderação de nível intermediário ou moderado, pois os efeitos de moderação acima de 0,40 raramente são observados na área.

As variáveis do modelo populacional foram configuradas como latentes, tendo cinco itens por fator (sem cargas cruzadas). As cargas fatoriais foram as seguintes: $0,90,0,90,0,50,0,50$ e $0,70(\mathrm{VD}) ; 0,95,0,95,0,45$, 0,45 e 0,70 (VMed); 0,80, 0,80, 0,60, 0,60 e 0,70 (VI); e $0,90,0,90,0,50,0,50$ e 0,70 (VMod). Assim, as cargas fatoriais, foram configuradas com média igual $0,70 \mathrm{e}$ não homogêneas entre os fatores (cenários comuns em Psicologia). Os itens de cada variável foram simulados como intervalares.

Para ambas as condições (efeito fraco e efeito moderado da VMod), buscou-se recuperar os parâmetros de efeito entre as variáveis por meio de três modelos: (a) slopes randômicos (com estimação da variância dos slopes da moderação); (b) interação latente (estimação de uma variável latente a partir da interação dos itens que compõem a VI e a VMod); e (c) path analysis (na qual as variáveis foram configuradas como observadas, e seus escores foram obtidos por meio da média dos itens).

Portanto, o estudo envolveu seis condições (dois tamanhos de efeito $\times$ três modelos). Foram simuladas 500 replicações de bancos de dados para cada condição de tamanho de efeito. Em cada banco de dados, o tamanho da amostra foi mantido fixo em 700 sujeitos simulados. Os bancos foram simulados por Monte Carlo, implementado no Mplus (versão 8.0). As análises de cada replicação também foram realizadas no Mplus, sendo automatizadas no pacote do R MplusAutomation (Hallquist \& Wiley, 2018).

\section{Resultados}

Os resultados do estudo de simulação são apresentados na Tabela 1. Os vieses, em porcentagem, representam a diferença entre o parâmetro da população (valor verdadeiro) e o valor estimado (ou recuperado pelo modelo). O viés foi calculado por meio da seguinte equação: [(parâmetro população - parâmetro estimado)/parâmetro população]. Assim, vieses altos indicam que o modelo estima valores de parâmetros distantes do verdadeiro, o que aponta para falha na modelagem.

Observa-se que o modelo de slope randômico foi capaz de recuperar os parâmetros com pouco viés (máximo de 6,57\%), ainda que a variância dos slopes seja pequena $(0,07)$. Esse resultado era esperado uma vez que os dados da população foram simulados para apresentarem, de fato, slopes randômicos. Ressalta-se, no entanto, que a diminuição do efeito, entre a primeira e a segunda condição (de 0,20 para 0,07$)$ foi acompanhada de significativo aumento de viés (de 1,7 para 6,57).

O modelo de interação latente recuperou adequadamente os efeitos quando a variância do slope randômico foi pequena $(0,07)$. Contudo, para amostras de slopes randômicos com maior variância $(0,20)$, o modelo de interações latentes apresenta vieses maiores.

O modelo de path analysis, com as variáveis calculadas a partir das médias dos itens, foi capaz de recuperar adequadamente os efeitos de moderação (em ambas as condições - efeito fraco e efeito moderado). Não obstante, a modelagem apresentou falhas nas estimações dos efeitos diretos (tanto da VD sobre a VMed, quanto da VMed-sobre a VI). Em todos os casos, os efeitos foram subestimados pela path analysis. Obviamente, os efeitos indiretos da mediação, neste caso, foram os mais afetados, uma vez que são calculados a partir da multiplicação dos efeitos diretos, bastante enviesados nessa modelagem.

As médias dos erros-padrão de estimativa dos parâmetros (EP) foram parecidas nas três modelagens: entre 0,03 e 0,06 para os efeitos diretos; e entre 0,05 e 0,06 para os efeitos de moderação. Esse resultado é importante, pois esse parâmetro é utilizado para concluir se um efeito é estatisticamente significativo ou não. Nesse sentido, 
espera-se que o valor de EP seja, no máximo, a metade do valor do efeito. Por exemplo, se o efeito é igual a 0,40, espera-se um EP menor do que 0,20. Assim, as três modelagens, considerando a média dos parâmetros estimados nas 500 replicações, apontaram para efeitos diretos e de moderação significativos.

Tabela 1

Diferenças entre os Parâmetros Populacionais Simulados e os Estimados por Modelos de Mediação Moderada

\begin{tabular}{|c|c|c|c|c|c|c|c|c|c|c|}
\hline \multirow{3}{*}{$\begin{array}{l}\text { Modelo } \\
\text { Parâmetro }\end{array}$} & \multicolumn{5}{|c|}{$\begin{array}{c}\text { moderação=0,40 \& mediação } \\
\text { (efeito indireto) }=0,20\end{array}$} & \multicolumn{5}{|c|}{$\begin{array}{c}\text { moderação=0,10 \& mediação } \\
\text { (efeito indireto)=0,05 }\end{array}$} \\
\hline & \multirow{2}{*}{ Pop. } & \multicolumn{2}{|c|}{ Efeito } & \multirow{2}{*}{$\begin{array}{c}\text { EP } \\
\text { Med }\end{array}$} & \multirow{2}{*}{$\begin{array}{l}\text { viés } \\
(\%)\end{array}$} & \multirow{2}{*}{ Pop. } & \multicolumn{2}{|c|}{ Efeito } & \multirow{2}{*}{$\begin{array}{c}\text { EP } \\
\text { Med }\end{array}$} & \multirow{2}{*}{$\begin{array}{l}\text { viés } \\
(\%)\end{array}$} \\
\hline & & Med & $\mathrm{DP}$ & & & & Med & $\mathrm{DP}$ & & \\
\hline \multicolumn{11}{|l|}{ Slope randômico ${ }^{a}$} \\
\hline slope direto VD \& VMed & 0,50 & 0,501 & 0,04 & 0,04 & 0,10 & 0,50 & 0,500 & 0,03 & 0,03 & 0,04 \\
\hline slope direto Vmed \& VI & 0,40 & 0,407 & 0,05 & 0,06 & 1,68 & 0,10 & 0,100 & 0,04 & 0,05 & 0,20 \\
\hline slope moderação (intercepto) & 0,40 & 0,399 & 0,06 & 0,06 & $-0,03$ & 0,10 & 0,099 & 0,05 & 0,05 & $-1,20$ \\
\hline variância do slope randômico & 0,20 & 0,203 & 0,09 & 0,09 & 1,70 & 0,07 & 0,075 & 0,06 & 0,06 & 6,57 \\
\hline \multicolumn{11}{|l|}{ Interação Latente ${ }^{a}$} \\
\hline slope direto VD \& VMed & 0,50 & 0,554 & 0,04 & 0,04 & 10,76 & 0,50 & 0,500 & 0,03 & 0,03 & 0,04 \\
\hline slope direto Vmed \& VI & 0,40 & 0,367 & 0,05 & 0,05 & $-8,20$ & 0,10 & 0,100 & 0,05 & 0,05 & 0,04 \\
\hline slope moderação (interação latente) & 0,40 & 0,370 & 0,05 & 0,05 & $-7,45$ & 0,10 & 0,100 & 0,04 & 0,05 & 0,10 \\
\hline variância do slope randômico & 0,20 & 0 (fixo) & & & & 0,07 & 0 (fixo) & & & \\
\hline \multicolumn{11}{|l|}{ Path Analysis com variáveis observadas } \\
\hline slope direto VD \& VMed & 0,50 & 0,439 & 0,03 & 0,03 & $-12,30$ & 0,50 & 0,425 & 0,03 & 0,03 & $-15,02$ \\
\hline slope direto Vmed \& VI & 0,40 & 0,336 & 0,04 & 0,04 & $-15,95$ & 0,10 & 0,083 & 0,04 & 0,04 & $-17,40$ \\
\hline slope moderação (interação) & 0,40 & 0,392 & 0,06 & 0,05 & $-1,93$ & 0,10 & 0,098 & 0,06 & 0,05 & $-2,20$ \\
\hline variância do slope randômico & 0,20 & 0 (fixo) & & & & 0,07 & 0 (fixo) & & & \\
\hline
\end{tabular}

Notas. A=as cargas fatoriais foram omitidas da tabela. Med=média dos parâmetros estimados nas 500 replicações; DP=desviopadrão dos parâmetros estimados nas 500 replicações; EP=erro-padrão de estimativa do parâmetro. Na path analysis as variáveis observadas foram calculadas por meio da média dos itens de cada fator.

\section{Exemplo com Dados Reais: Aprendizagem e Desempenho no Trabalho}

Para ilustrar as diferenças entre as modelagens de mediação moderada foi utilizado um banco de dados reais de uma pesquisa no contexto da Psicologia Organizacional. O banco contém dados de 750 trabalhadores brasileiros oriundos de empresas em processo de mudança. Nesse modelo, buscou-se avaliar se o engajamento no trabalho poderia mediar a relação entre suporte à aprendizagem e desempenho no trabalho, bem como avaliar se a intensidade da mudança poderia moderar essa relação indireta. Para tanto, foram utilizados os instrumentos: 1. Escala Utrecht de Engajamento no Trabalho (UWES-9), composta por nove itens (exemplo, "Em meu trabalho, sinto-me repleto de energia”); 2. Escala de Suporte à Aprendizagem no Contexto de Mudança Organizacional (ESACoM), composta por 17 itens (exemplo, "A busca de novas aprendizagens é valorizada pela organização no processo de mudança"); 3. Escala de Autoavaliação de Desempenho no Trabalho (EGDT-r), composta por 10 itens (exemplo, "Busco novas soluções para problemas que possam surgir em meu trabalho”); 4.
Escala de Intensidade da Mudança Organizacional, composta por quatro itens (exemplo, "O processo de mudança levou à modificação na estrutura organizacional”). Todos os instrumentos eram de autorelato e respondidos por meio de uma escala do tipo Likert de cinco pontos, ancorados entre 1 (Nunca) e 5 (Sempre).

Para avaliar o modelo, primeiramente, testou-se a estrutura latente dos escores dos instrumentos, que exige fatores minimamente independentes (i.e., os fatores devem apresentar validade discriminante). Caso contrário, uma parte significativa do um efeito de uma variável sobre a outra poderia ser atribuída às correlações entre as dimensões. Para avaliar essa hipótese, buscou-se comparar as correlações entre as variáveis latentes e as variâncias médias extraídas (VME) (Valentini \& Damásio, 2016). O esperado seria que a maior parte da variância modelada pudesse ser atribuída às cargas fatoriais e não às correlações entre os fatores, caso contrário não se observaria evidências de validade discriminante. As correlações entre os fatores latentes, coeficientes de determinação (i.e. cargas fatoriais ao quadrado) e as VMEs são apresentadas na Tabela 2 . 
Tabela 2

Cargas Fatoriais, Coeficientes de Determinação e Correlações Latentes entre os Fatores

\begin{tabular}{lccccc}
\hline & VME & 1 & 2 & 3 & $\mathbf{0}$ \\
\hline 1. Suporte à Aprendizagem & 0,63 & & $\mathbf{0 , 2 3}$ & $\mathbf{0 , 4 7}$ & $\mathbf{0 , 1 8}$ \\
2. Intensidade da Mudança & 0,49 & 0,48 & & $\mathbf{0 , 2 6}$ & $\mathbf{0 , 1 4}$ \\
3. Engajamento & 0,69 & 0,69 & 0,51 & $\mathbf{0 , 3 7}$ \\
4. Desempenho & 0,44 & 0,42 & 0,37 & 0,61 & \\
\hline
\end{tabular}

Nota. VME=Variância média extraída; na diagonal superior, são apresentados os coeficientes de determinação (correlação elevada ao quadrado); na diagonal inferior, são apresentadas as correlações estimadas entre as variáveis latentes. As cargas fatoriais foram omitidas

Observa-se, na Tabela 2 que as correlações entre engajamento, desempenho e suporte à aprendizagem foram altas. No entanto, os coeficientes de determinação foram inferiores às VMEs (suporte \& engajamento, VME $=0,63$ e 0,69 ; coeficiente de determinação $=0,47$; engajamento \& desempenho, $\mathrm{VME}=0,69$ e 0,44 ; coeficiente de determinação $=0,37)$. Essa análise pode ser estendida às demais variáveis, pois todos os coeficientes de determinação foram inferiores às VMEs, indicando que a maior parte da covariância dos itens pode ser atribuída aos fatores e não às correlações entre eles. Em outras palavras, esses resultados apontam para evidências de que os fatores são minimamente independentes entre si.

Tendo assegurada a estrutura, o passo seguinte foi testar modelos de relações. Para a ilustração, na primeira etapa, envolvendo apenas os efeitos indiretos de mediação, os parâmetros foram estimados por Maximum Likelihood e todas as variáveis foram configuradas como latentes no contexto da modelagem por equações estruturais.

No primeiro passo, avaliou-se a relação direta entre o suporte à aprendizagem e o desempenho no trabalho. Esse efeito foi estatisticamente significativo (B, coeficiente não padronizado $=0,45, \mathrm{EP}=0,05 ; \beta=0,41)$. No segundo passo, inseriu-se a variável mediadora engajamento no trabalho. Na presença de tal VMed, a relação direta entre suporte e desempenho não foi estatisticamente significativa $(\mathrm{B}=0,03, \mathrm{EP}=0,06 ; p=0,61)$. Observou-se que os demais efeitos foram estatisticamente significativos $(p<0,01)$, a saber: efeito direto entre o suporte e o engajamento (VI e VMed) teve $(\mathrm{B}=0,88, \mathrm{EP}=0,07 ; \beta=0,67)$; e efeito direto entre o engajamento e o desempenho (VMed e VD; $\mathrm{B}=0,54, \mathrm{EP}=0,08 ; \beta=0,58)$. O efeito indireto (i.e. mediação do engajamento sobre a relação entre suporte e desempenho) foi estimado por meio de 500 reamostragens bootstrap e também foi estatisticamente significativo $(B=0,48, E P=0,07 ; \beta=0,38)$. Assim, neste passo, concluiu-se pela mediação total, pois: (a) o efeito direto entre VI e VD desaparece na presença da mediadora; (b) os efeitos diretos entre VI e VMed e entre VMed e VD foram significativos; e (c) o efeito indireto entre VI, VMed e VD também foi significativo.

No terceiro passo, inseriu-se no modelo a variável moderadora, intensidade da mudança (VMod) para testar se a relação entre suporte à aprendizagem (VI) e engajamento (VMed) poderia variar de acordo com tal condicionante. Como o engajamento é uma variável mediadora, o modelo final envolve a moderação do efeito direto entre VI e VMed, bem como a moderação do efeito indireto entre VI-VMed-VD. Esse é o modelo final que está sendo testado para ilustrar os argumentos do presente artigo, por isso, são apresentadas diferentes formas de configurá-lo, relatando-se o impacto disso na estimação dos parâmetros.

Os formatos testados envolveram: Modelo 1 - variáveis configuradas como observadas a partir das médias dos itens de cada escala; Modelo 2 - variáveis configuradas como observadas a partir dos escores latentes (nesse formato, foram realizadas análises fatoriais confirmatórias para cada escala e foram salvos, no banco de dados, os escores fatoriais); Modelo 3A - variáveis configuradas como latentes (esse formato é um modelo de equações estruturais completo, no qual os parâmetros dos itens são estimados simultaneamente aos coeficientes de regressão entre as variáveis latentes), e moderação configurada por meio de variável de interação latente; e Modelo 3B - modelo configurado com slopes randômicos, além de variáveis latentes. Destaca-se que, neste último modelo, a moderação é decomposta entre efeito e variância residual. Os efeitos dessas modelagens são apresentados na Tabela 3 .

Em todos os modelos, a conclusão foi a mesma: a intensidade da mudança moderou negativamente a relação entre suporte à aprendizagem (VI) e engajamento (VMed). Tem-se, portanto, uma mediação moderada que indica uma combinação entre um modelo com efeitos indiretos e com interação.

Apesar de a conclusão ser a mesma em todos os modelos testados, é preciso destacar algumas diferenças entre as configurações testadas. A primeira envolve os erros-padrão de estimativa (EP). No Modelo 1, com variáveis observadas (pelo cálculo da média dos itens), a proporção do EP sobre o efeito de mediação foi maior do que os demais $(0,03 / 0,06=0,5)$. Isso era esperado, uma vez que tal configuração desconsidera o erro de medida e assume que todos os itens apresentam o mesmo peso (i.e., a mesma carga fatorial) no cômputo da variável. Assim, o EP passa a ser inflado, o que diminui o poder 
estatístico da análise (i.e., poder da análise em detectar, na amostra, os efeitos significativos quando eles de fato existem na população). Contudo, cumpre o registro de que essa inflação do EP, nos dados utilizados foi pequena.

A segunda diferença diz respeito aos tamanhos de efeito. Os modelos que ponderam as cargas fatoriais
$(2,3 \mathrm{~A}$ e $3 \mathrm{~B})$ apresentaram padrões de efeitos de moderação e indireto semelhantes (sendo o efeito de moderação aproximadamente a metade do efeito indireto). Mesmo no Modelo 2, - configurado com variáveis observadas a partir dos escores fatoriais - os efeitos são semelhantes aos modelos "puramente" latentes (3A e 3B).

Tabela 3

Parâmetros da Moderação dos Efeitos Indiretos Estimados por Modelos com Variáveis Observadas e Latentes

\begin{tabular}{|c|c|c|c|c|c|c|}
\hline \multirow[b]{2}{*}{ Modelos } & \multicolumn{4}{|c|}{$\begin{array}{c}\text { Efeitos } \\
\text { não padronizados (B) }\end{array}$} & \multicolumn{2}{|c|}{$\begin{array}{c}\text { Efeitos } \\
\text { padronizados }(\beta)\end{array}$} \\
\hline & $\begin{array}{c}\text { Efeito } \\
\text { Moderação } \\
\text { B (EP);p }\end{array}$ & $\begin{array}{l}\text { Variância } \\
\text { do Efeito } \\
\text { Moderação } \\
\text { B (EP);p }\end{array}$ & $\begin{array}{c}\text { Relação } \\
\text { VI-VMed } \\
\text { B (EP) }\end{array}$ & $\begin{array}{c}\text { Efeito } \\
\text { Indireto } \\
\text { Mediação } \\
\text { B (EP) }\end{array}$ & $\begin{array}{c}\text { Efeito } \\
\text { Moderação }\end{array}$ & $\begin{array}{l}\text { Efeito } \\
\text { Indireto }\end{array}$ \\
\hline $\begin{array}{l}\text { 1. Variáveis observadas } \\
\text { (médias dos itens) }\end{array}$ & $\begin{array}{c}-0,06(0,03) \\
p=0,02\end{array}$ & - & $0,71(0,10)$ & $0,19(0,09)$ & $-0,36$ & $-0,27$ \\
\hline $\begin{array}{l}\text { 2. Variáveis observadas } \\
\text { (escores fatoriais) }\end{array}$ & $\begin{array}{c}-0,11(0,03) \\
p=0,001\end{array}$ & - & $0,55(0,03)$ & $0,35(0,04)$ & $-0,11$ & $-0,39$ \\
\hline $\begin{array}{l}\text { 3A. Variáveis latentes - modelo } \\
\text { com interação latente }\end{array}$ & $\begin{array}{c}-0,25(0,07) \\
p=0,001\end{array}$ & - & $0,74(0,07)$ & $0,39(0,09)$ & $-0,17$ & $-0,31$ \\
\hline 3B. Slope randômico & $\begin{array}{c}-0,24(0,08) \\
p=0,002\end{array}$ & $\begin{array}{c}0,06(0,33) \\
p=0,86\end{array}$ & $\begin{array}{l}0,76(0,13) \\
\text { Média }^{a}\end{array}$ & $0,39(0,14)$ & $b$ & $b$ \\
\hline
\end{tabular}

Nota. ${ }^{a}=$ Por se tratar de um modelo de efeitos randômicos, a relação direta entre VI e VMed apresenta média e variância estimadas. $b=$ no modelo, os slopes se tornam uma variável, sua variância estimada é utilizada na padronização e, consequentemente, apresentam métrica distinta dos demais

O Modelo 3B apresenta métrica bastante distinta dos demais, pois considera a variância do efeito de slope randômico, em si, para o cômputo da padronização do efeito. Como essa variância tende a ser bem menor do que a de uma variável latente, o efeito de moderação é padronizado em uma escala acima dos demais (nesse caso, o valor padronizado do efeito de moderação foi igual a $-0,71$ e do efeito indireto foi igual a 1,32). Mesmo assim, ao compararmos os efeitos não padronizados (B) dos Modelos 3A e 3B, percebe-se valores muito semelhantes, indicando que, se a padronização dos efeitos do Modelo 3B pudesse ser realizada com base na variância das latentes, em vez da variância dos slopes, os efeitos padronizados desses modelos tenderiam a valores muito parecidos. Observa-se ainda que os efeitos padronizados do Modelo 1 são consideravelmente distintos dos demais. O tamanho de efeito da moderação, por exemplo, é cerca do dobro dos outros modelos.

A terceira diferença está associada aos modelos 3A e 3B. Tecnicamente, a variância residual dos slopes randômicos (ou resíduo do efeito de moderação) é o parâmetro que distingue esses modelos. Embora o 3B possa ser considerado mais "elegante", o referido resíduo não foi estatisticamente significativo no banco de dados do exemplo. Realizou-se um teste adicional com o Modelo
3B, fixando a variância residual dos slopes randômicos em zero (teste não apresentado na Tabela 3). Esse teste retornou exatamente os mesmos valores de parâmetros (incluindo o valor de ajuste log likelihood) do Modelo 3A. Assim, quando os slopes randomizados não apresentarem variância residual diferente de zero (como no exemplo com os dados reais aqui apresentados), o modelo de slopes randômicos $(3 \mathrm{~B})$ equivale ao modelo de interação latente (3A). Em suma, o modelo de slopes randômicos permite decompor a interação (moderação) entre efeito e resíduo. Mas essa "superioridade" do modelo se aplica apenas quando a variância dos resíduos dos efeitos randômicos for significativa.

\section{Discussão}

Fenômenos psicológicos são complexos e difíceis de serem compreendidos por simples relações diretas. O presente trabalho buscou apresentar e comparar modelos de moderação e mediação simultâneas capazes de aprofundar a compreensão da dinâmica das relações entre variáveis psicológicas. Para tanto, uma parte desta pesquisa envolveu um estudo de simulação de dados. Os modelos latentes foram superiores na recuperação de parâmetros de modelos complexos que envolvem, 
simultaneamente, a estrutura de escalas (com fatores, itens, cargas fatoriais e demais parâmetros dos itens) e as relações entre variáveis, se comparados à modelagem de path analysis. Essa conclusão também pode ser, hipoteticamente, estendida aos testes de mediação e moderação por regressões, uma vez que as variáveis também são configuradas como observadas.

Os vieses dos parâmetros estimados por path analysis podem ser atribuídos, em parte, à sua simplicidade, que desconsidera o erro de medida das variáveis (Fornell \& Larcker, 1981). No presente estudo de simulação, o problema pode ter sido agravado porque as cargas fatoriais não serem homogêneas (o que é algo comum em variáveis da Psicologia). Como a análise de mediação moderada por path analysis (e por regressão também) assume, implicitamente, que as cargas são todas iguais, as diferentes cargas fatoriais do modelo verdadeiro podem ter impactado significativamente na modelagem.

De maneira geral, a modelagem com variáveis observadas tendeu a subestimar os efeitos da mediação, mas não apresentou viés significativo na estimação dos efeitos de moderação. Tais resultados são coerentes com os estudos anteriores (Cheung \& Lau, 2008, 2017; Hoyle \& Kenny, 1999; Little et al., 2009; Muthén \& Asparouhov, 2003, 2015), que também apontaram para vieses maiores nos efeitos estimados por modelagens para variáveis observadas (como path analysis e regressões). Tais resultados têm implicações importantes, pois os efeitos de um programa ou de uma intervenção, por exemplo, poderiam ser subdimensionados, caso estimados por modelagens de regressão ou por path analysis. Em que pese a superioridade dos modelos latentes, destaca-se que todas as modelagens foram capazes de detectar os efeitos de mediação e moderação.

No que se refere à principal contribuição original deste estudo, a comparação entre os modelos de interações latentes e de slopes randômicos indicou que ambos são capazes de estimar os efeitos de moderação e mediação de maneira adequada (com baixo viés), quando esses efeitos e a variância dos slopes são pequenos. Contudo, quando a variância dos slopes randômicos é maior, o modelo de interação latente apresenta vieses relevantes de estimação. Esse resultado pode ser explicado pela diferença entre o modelo de slopes randômicos e o de interações latentes ser justamente o parâmetro de variância dos slopes: estimado no primeiro e fixado em zero no segundo. Quando essa variância se aproxima de zero, os dois modelos são praticamente equivalentes e recuperam os parâmetros verdadeiros com eficiência. No entanto, com o aumento da variância dos slopes, a distância dos modelos fica mais evidente e o modelo de interações latentes torna-se menos indicado. Ainda assim, mesmo neste cenário extremo, o modelo de interações latentes apresentou performance superior ao de path analysis.

Ressalta-se, ainda, a leve redução da performance do modelo de slopes randômicos na sua capacidade de estimar variância dos slopes, quando essa variância é reduzida de 0,20 para 0,07 . Isso pode estar associado ao menor poder estatístico, visto que o tamanho de amostra foi mantido constante $(n=700)$. Assim, recomenda-se cautela na utilização desse modelo quando a variância dos slopes for pequena. Se esse for o caso, sugere-se também aumentar o tamanho amostral.

Os resultados com dados reais apontaram para conclusões semelhantes às obtidas com as simulações: os modelos latentes parecem convergir melhor se comparados aos modelos estimados a partir da média dos itens. Nesse último, os parâmetros dos itens são assumidos como iguais (invariantes) entre os itens (mesmo que implicitamente). Consequentemente, os escores dos indivíduos e as variâncias de tais escores desconsideram eventuais diferenças de dificuldade, discriminação e resíduos dos itens. Em resumo, no banco de dados do exemplo, a utilização da média para calcular as variáveis (Modelo 1) parece superestimar o tamanho do efeito da moderação e subestimar o efeito indireto da mediação do engajamento no trabalho.

Ainda sobre a testagem realizada com dados reais, cabe uma discussão sobre a interpretação de seus resultados. Nos quatro modelos testados, a intensidade da mudança moderou negativamente a relação entre suporte à aprendizagem (VI) e engajamento (VMed), configurando uma mediação moderada que indica uma combinação entre um modelo com efeitos indiretos e com interação. Considerando as variáveis utilizadas no exemplo, os resultados obtidos podem ser explicados da seguinte forma: o suporte à aprendizagem apresenta um efeito indireto sobre o desempenho, por meio do seguinte caminho: o suporte à aprendizagem conduz ao engajamento no trabalho que, por sua vez, leva a melhorias no desempenho laboral. A relação entre essas variáveis sofre a interação da intensidade da mudança, de tal sorte que a força que o suporte à aprendizagem exerce sobre o engajamento é reduzida em um contexto de intensa mudança organizacional. Em outras palavras, a intensidade da mudança atua como uma condicionante para a relação entre o suporte à aprendizagem e o engajamento no trabalho.

Os modelos com dados reais apresentaram diferenças sutis, mas todos permitem chegar às conclusões principais se há ou não efeito de mediação, moderação e mediação moderada. Do ponto de vista metodológico, considerando as diferenças observadas nas análises, sugere-se, sempre que possível, utilizar modelos de interação latente ou de slopes randômicos. E, quando isso não for possível (por limitações da amostra, dos instrumentos ou de familiaridade com as modelagens), o pesquisador não deve deixar de testar modelos que combinem mediação e moderação. Por um lado, esses modelos mais complexos podem fornecer explicações importantes para as pesquisas em Psicologia; e, por 
outro, as análises revelaram que os quatro modelos testados permitem identificar os resultados em termos de relações que envolvem VI, VD, VMod e VMed.

$\mathrm{O}$ presente estudo tem limitações importantes. A primeira delas diz respeito ao limitado número de condições testadas nas simulações. Assim, sugere-se que estudos futuros avaliem diferentes tamanhos amostrais e tamanhos de efeitos das relações entre as variáveis. Além disso, os vieses de resposta, muito comum em pesquisas de autorrelato, não foram testados, nem nos dados simulados, nem nos dados reais. Nesse sentido, estudos futuros podem avaliar o impacto da aquiescência e da desejabilidade social, por exemplo, nos testes de moderação e mediação.
Por fim, os resultados da presente pesquisa corroboram os estudos anteriores e sugerem, sempre que possível, o uso de modelagem latente para testar modelos complexos. Além disso, é possível indicar um guia, em passos, para pesquisadores. Inicialmente, busque avaliar os dados por meio do modelo de slopes randômicos. Avalie, cuidadosamente, a variância desses slopes. Em seguida, caso essa variância não seja estatisticamente significativa, modele com interações latentes e compare os resultados com os do modelo anterior. Por fim, como um terceiro passo, caso não seja possível utilizar modelagem latente, configure as variáveis como observadas, mas preste atenção nos tamanhos de efeitos diretos, que podem, neste caso, ficarem subdimensionados.

\section{Referências}

Abbad, G., \& Torres, C.V. (2002). Regressão múltipla stepwise e hierárquica em psicologia organizacional: Aplicações, problemas e soluções. Estudos em Psicologia (Natal), 7(Ed. Especial), 19-29. doi: 10.1590/S1413-294X2002000300004.

Aguinis, H., Edwards, J. R., \& Bradley, K. J. (2017). Improving our understanding of moderation and mediation in strategic management research. Organizational Research Methods, 20(4), 665-685. doi: 10.1177/1094428115627498

Baron, R. M., \& Kenny, D. A. (1986). The moderator-mediator variable distinction in social psychological research: Conceptual, strategic, and statistical considerations. Journal of Personality and Social Psychology, 51, 1173-1182.

Bollen, K. A. (1989). Structural equations with latent variables. New York: Wiley.

Cheung, G.W., \& Lau, R.S. (2008). Testing mediation and suppression effects of latent variables: Bootstrapping with structural equation models. Organizational Research Methods, 11(2), 296-325. doi: 10.1177/1094428107300343.

Cheung, G. W., \& Lau, R. S. (2017). Accuracy of parameter estimates and confidence intervals in moderated mediation models: A comparison of regression and latent moderated structural equations. Organizational Research Methods, 20(4), 746-769. doi: 10.1177/1094428115595869

Cohen, J., \& Cohen, P. (1983). Multiple regression/correlation for the behavioral sciences. Mahwah, NJ: Lawrence Erlbaum Associates Publishers.

Donaldson, S. I. (2001). Mediator and moderator analysis in program development. Em S. Sussman (Ed.). Handbook of program development for health behavior research and practice (pp. 470-496). Thousand Oaks, CA: Sage.

Edwards, J. R., \& Lambert, L. S. (2007). Methods for integrating moderation and mediation: A general analytical framework using moderated path analysis. Psychological Methods, 12(1), 1-22. doi: 10.1037/1082-989X.12.1.1

Fiske, S., Kenny, D. A., \& Taylor, S. E. (1982). Structural models for the mediation of salience effects. Journal of Experimental Social Psychology, 18, 105-127. doi: 10.1016/0022-1031(82)90046-4.

Fornell, C., \& Larcker, D. F. (1981). Structural equation models with unobservable variables and measurement error: Algebra and statistics. Journal of Marketing Research, 18(3), 382-388. doi: 10.2307/3150980.

Hallquist, M. N., \& Wiley, J. F. (2018). MplusAutomation: An R package for facilitating large-scale latent variable analyses in Mplus. Structural Equation Modeling: A Multidisciplinary Journal, 25(4), 621-638. doi: 10.1080/10705511.2017.140233

Hayes, A. F. (2009). Beyond baron and kenny: Statistical mediation analysis in the new millennium. Communication Monographs, 76(4), 408420. doi: $10.1080 / 03637750903310360$

Hayes, A. F. (2013). Introduction to mediation, moderation, and conditional process analysis: A regression-based approach. New York, NY: Guilford Press.

Hoyle, R. H., \& Kenny, D. A. (1999). Sample size, reliability, and tests of statistical mediation. Em R. H. Hoyle (Ed.), Statistical strategies for small sample research (pp. 195-222). Thousand Oaks, CA: Sage.

Hyman, H. H. (1955). Survey Design and Analysis. New York and Glencoe, IL: The Free Press.

James, L. R., \& Brett, J. M. (1984). Mediators, moderators and tests for mediation. Journal of Applied Psychology, 69, 307-321. doi: 10.1037/00219010.69.2.307.

Kenny, D. A. (2008). Reflections on mediation. Organizational Research Methods, 11(2), 353-358. doi: 10.1177/1094428107308978

Klein, A., \& Moosbrugger, H. (2000). Maximum likelihood estimation of latent interaction effects with the LMS method. Psychometrika, 65(4), 457-474. doi: 10.1007/BF02296338

Klein, A., \& Muthén, B. O. (2002). Quasi maximum likelihood estimation of structural equation models with multiple interaction and quadratic effects. Unpublished manuscript, Graduate School of Education, University of California, Los Angeles.

Little, T. D., Bovaird, J. A., \& Widaman, K. F. (2009). On the merits of orthogonalizing powered and product terms: Implications for modeling interactions among latent variables. Structural Equation Modeling: A Multidisciplinary Journal, 13(4), 497-519, doi: 10.1207/ s15328007sem1304_1

Little, T. D., Card, N. A., Bovaird, J. A., Preacher, K. J., \& Crandall, C. S. (2007). Structural Equation Modeling of mediation and moderation with contextual factors. Em T. D. Little, J. A. Bovaird \& N. A. Card (Eds.), Modeling contextual effects in longitudinal studies (pp. 207-230). New York, NY: Lawrence Erlbaum Associates

MacKinnon, D. P. (2008). Introduction to statistical mediation analysis. New York, NY: Lawrence Erlbaum Associates. 
MacKinnon, D. P., Lockwood, C. M., Hoffman, J. M., West, S. G., Sheets, V. (2002). A comparison of methods to test mediation and other intervening variable effects. Psychological Methods, 7(1), 83-104. doi: 10.1037/1082-989X.7.1.83.

Morgan-Lopez, A. A., \& MacKinnon, D. P. (2006). Demonstration and evaluation of a method for assessing mediated moderation. Behavior Research Methods, 38(1), 77-87. doi: 10.3758/BF03192752.

Muthén, B. (2012). Latent variable interactions (Unpublished manuscript). Recuperado de http://www.statmodel.com/download/LV/ Interaction.pdf

Muthén, B. \& Asparouhov, T. (2003). Modeling interactions between latent and observed continuous variables using maximum-likelihood estimation in Mplus. Mplus Web Notes, 6. Recuperado de http://statmodel.com/examples/webnote.shtml

Muthén, B., \& Asparouhov, T. (2015). Causal effects in mediation modeling: An introduction with applications to latent variables. Structural Equation Modeling: A Multidisciplinary Journal, 22, 12-23. doi: 10.1080/10705511.2014.935843

Preacher, K. J., Rucker, D. D., \& Hayes, A. F. (2007). Addressing moderated mediation hypotheses: Theory, methods, and prescriptions. Multivariate Behavioral Research, 42(1), 185-227. doi: 10.1080/00273170701341316

Rozeboom, W. W. (1956). Mediation variables in scientific theory. Psychological Review, 63(4), 249-264. doi: 10.1037/h0043718.

Trautwein, U., Marsh, H. W., Nagengast, B., Lüdtke, O., Nagy, G., \& Jonkmann, K. (2012). Probing for the multiplicative term in modern expectancy-value theory: A latent interaction modeling study. Journal of Educational Psychology, 104(3), 763-777. doi: 10.1037/a0027470

Valentini, F., \& Damásio, B. F. (2016). Variância média extraída e confiabilidade composta: Indicadores de precisão. Psicologia: Teoria e Pesquisa, 32(2), 1-7. doi: 10.15900102-3772e322225

Wu, A. D., \& Zumbo, B. D. (2008). Understanding and using mediators and moderators. Social Indicators Research, 87, 367-392. doi: 10.1007/ s11205-007-9143-1

Zhang, Q., \& Wang, L. (2017). Moderation analysis with missing data in the predictors. Psychological Methods, 22(4), 649-666. doi: 10.1037/ met0000104

Zhao, X., Lynch Jr, J. G., \& Chen, Q. (2010). Reconsidering Baron and Kenny: Myths and truths about mediation analysis. Journal of Consumer Research, 37(2), 197-206. doi: 10.1086/65125

\section{Sobre os autores}

Felipe Valentini é doutor em Psicologia pela Universidade de Brasília (UnB). Atualmente, é Professor no Programa de Pós-Graduação Stricto Sensu em Psicologia da Universidade São Francisco, Campinas. Bolsista de Produtividade CNPq.

Luciana Mourão é doutora em Psicologia pela Universidade de Brasília (UnB). Atualmente, é Professora no Programa de Pós-Graduação Stricto Sensu em Psicologia da Universidade Salgado de Oliveira, Niterói. Bolsista de Produtividade CNPq.

Vithor Rosa Franco é mestre em Psicologia pela Universidade de Brasília (UnB). Atualmente, é doutorando do Programa de Pós-Graduação em Psicologia Social, do Trabalho e das Organizações da UnB. 